\title{
High-frequency wavepackets in turbulent jets
}

\author{
André V. G. Cavalieri*and Kenzo Sasaki ${ }^{\dagger}$ \\ Instituto Tecnológico de Aeronáutica, São José dos Campos, SP, 12228-900, Brazil \\ Oliver Schmidt ${ }^{\ddagger}$ and Tim Colonius ${ }^{\S}$ \\ California Institute of Technology, Pasadena, CA, USA \\ Peter Jordan \\ Institut Pprime, CNRS - Université de Poitiers - ENSMA, 86000 Poitiers, France \\ Guillaume A. Brès"l \\ Cascade Technologies Inc., Palo Alto, CA 94303, USA
}

\begin{abstract}
Wavepackets obtained as solutions of the flow equations linearised around the mean flow have been shown in recent work to yield good agreement with the amplitudes and phases of turbulent fluctuations in jets. Compelling agreement has been demonstrated up to Strouhal numbers, $S t \approx 1$. We extend the range of validity of wavepacket models to higher values, $1.0<S t<4.0$, by comparing Parabolised Stability Equation solutions with well resolved large-eddy simulation data. The initial growth rates of the high-frequency fluctuations continue to be well predicted, but saturation occurs earlier and agreement with simulation begins to deteriorate upstream of the end of the potential core of the jet. Results show that near-nozzle dynamics for a broad range of frequencies can be modelled using linearised models, which capture well the spatial growth of Kelvin-Helmholtz wavepackets for all the studied Strouhal numbers.
\end{abstract}

\section{Introduction}

Predicting and reducing the noise radiated by turbulent jets are two challenging long-term technological problems. For commercial aircraft noise regulations are the driving factor; where military aircraft are concerned hearing loss of personnel is pushing the military to search for noise-reduction solutions. ${ }^{1}$ The conception of effective strategies for jet-noise control is contingent on a thorough understanding of the underlying flow physics, and on the availability of associated reduced-order models. In addition to the lower computational expense that such models present, in comparison to large-eddy or direct-numerical simulations, they have the added advantage of providing clear physical insight regarding the mechanisms of sound generation. ${ }^{2,3}$

The coherent part of the velocity field of high-Reynolds-number turbulent jets comprises a wavepacket: a hydrodynamic wave with amplitude growth due to the Kelvin-Helmholtz instability of the shear layer, followed by saturation and decay. Such structures are characterised by low azimuthal wavenumber and significant coherence in both radial and axial directions. While their contribution to the turbulent kinetic energy is small, they dominate sound radiation to low polar angles (measured with respect to the jet axis). ${ }^{4}$

Wavepacket modelling is based on a linearisation of the Navier-Stokes system using the mean field as a base flow. This linearised system neglects, at least explicitly, non-linear interactions between wavepackets and turbulence; such interactions can only occur via the mean flow, which is mainly established by Reynolds

\footnotetext{
*Assistant Professor, Divisão de Engenharia Aeronáutica, Instituto Tecnológico de Aeronáutica, AIAA Member.

${ }^{\dagger}$ PhD student, Divisão de Engenharia Aeronáutica, Instituto Tecnológico de Aeronáutica, AIAA Member.

$\ddagger$ Post-doctoral scholar, California Institute of Technology, Pasadena, USA

$\S$ Professor, California Institute of Technology, Pasadena, USA

I Research Scientist, CNRS, Poitiers, France

"ISenior Research Scientist, Cascade Technologies Inc., Palo Alto, USA
} 
stresses associated with the energy-containing turbulent eddies. ${ }^{4,5}$ Such models rely on two hypotheses: a scale separation between wavepackets and turbulent eddies (necessary for neglect of the said non-linear interactions) and low fluctuation amplitudes (the requirement for linearisation). The validity of such models is usually demonstrated a posteriori by comparison with experimental data; in the past this comparison involved forced jets, ${ }^{6,7}$ more recently data from unforced jets has been used..$^{8-10}$

The agreement observed between experiment and wavepacket-based reduced order models, particularly in regions upstream of the end of the jet potential core, has motivated several studies. In addition to the round subsonic jets studied in the work cited above, linear wavepacket models have been used to model coaxial $^{11,12}$ and supersonic jets; ${ }^{13-16}$ for the latter, wavepackets can be readily extended to the acoustic field to obtain Mach-wave radiation. Recently, Sinha et al. ${ }^{16}$ compared wavepacket model predictions to results from large-eddy simulations of cold and heated supersonic jets, and found encouraging agreement for the peak far-field radiation. Sound radiation by wavepackets in subsonic jets is more subtle, and depends on details of the amplitude envelope ${ }^{17}$ and on the two-point coherence. ${ }^{18}$ These mechanisms are the subject of ongoing research, which considers either simplified, kinematic sound-source models ${ }^{19-21}$ or the coupling of dynamic wavepacket models with an acoustic analogy. ${ }^{22-25}$

The purpose of the work described here is to investigate the limits of the underlying hypotheses in wavepacket models by comparing solutions of the linear Parabolised Stability Equations (PSE) with experimental data for a turbulent subsonic jet previously studied by this group. ${ }^{10}$ That work has shown that the axisymmetric and first helical modes of the velocity field correspond to linear wavepackets, close agreement being observed between PSE and experiment for Strouhal numbers ranging from 0.3 to 0.9 , and for axial stations ranging from the nozzle exit plane to the end of the potential core of the jet. The existence of wavepacket structures at higher Strouhal numbers has not yet, to the best of our knowledge, been demonstrated.

Higher-St wavepackets are particularly relevant where the effect of nozzle conditions on sound radiation is concerned. The noise spectrum is changed over a broad range of frequencies when either nozzle contraction ${ }^{26}$ or boundary-layer thickness at the nozzle exit ${ }^{27}$ are modified. The interpretation of results of such studies, currently a subject of some controversy, would benefit from an extension of the results of wavepacket theory to higher frequencies.

The above issues, and the fact that jet noise comprises a broad frequency spectrum, whose higher frequencies have a greater relative importance due to the sensitivity of the human ear, make the extension of wavepacket modelling to $S t>1.0$ of considerable practical interest. In what follows we will examine how PSE-based wavepacket models behave for Strouhal numbers up to 4, with a comparison to simulation data of a Mach 0.9 jet.

\section{Mathematical model}

The PSE system is obtained by considering the flow variables, $\mathbf{q}=\left[u_{x}, u_{r}, u_{\theta}, \rho, T\right]^{T}$, to be decomposed into a mean axisymmetric component, $\overline{\mathbf{q}}(\mathbf{x})$, and a fluctuation, $\mathbf{q}^{\prime}(\mathbf{x}, t)$, that can be represented using normal modes in azimuth and time, and a slow variation in the axial direction,

$$
\mathbf{q}^{\prime}(\mathbf{x}, t)=\hat{\mathbf{q}}(x, r) \mathrm{e}^{\mathrm{i} \int^{x} \alpha\left(x^{\prime}\right) \mathrm{d} x^{\prime}} \mathrm{e}^{\mathrm{i} m \theta} e^{-\mathrm{i} \omega t},
$$

where $\alpha=\alpha_{r}+\mathrm{i} \alpha_{i}$ is the complex axial wavenumber whose imaginary part is related to exponential growth or decay of disturbances in $x$. Slow variations in $x$ are assumed for both $\hat{\mathbf{q}}$ and $\alpha$.

Introducing this Ansatz into the linearised, compressible Euler, energy and continuity equations, a matrix system is obtained,

$$
(A(\overline{\mathbf{q}})+B(\overline{\mathbf{q}}, \alpha, \omega)) \hat{\mathbf{q}}+C(\overline{\mathbf{q}}) \frac{\partial \hat{\mathbf{q}}}{\partial x}+D(\overline{\mathbf{q}}) \frac{\partial \hat{\mathbf{q}}}{\partial r}=0 .
$$

Details of the derivation may be found elsewhere. ${ }^{2,28} \mathrm{~A}$ further condition is necessary in order to eliminate the ambiguity between $\hat{\mathbf{q}}$ and $\alpha$, as the axial growth could be described by either the shape function $\hat{\mathbf{q}}$ and/or the complex amplitude $\mathrm{e}^{\mathrm{i} \int \alpha\left(x^{\prime}\right) d x^{\prime}}$. We follow the normalisation proposed in Herbert et al. ${ }^{28}$ which removes the exponential axial dependence from the shape function by imposing

$$
\int_{0}^{\infty} \hat{\mathbf{q}} \frac{\partial \hat{\mathbf{q}} *}{\partial x} r \mathrm{~d} r=0
$$


Discretisation of equations 2 and 3 permits numerical solution for $\hat{\mathbf{q}}$ and $\alpha .^{9}$ The radial direction is discretised using Chebyshev polynomials, providing a pseudo-spectral accuracy which is desirable on account of the high Strouhal-numbers considered. Axial derivatives are computed using finite differences, and spatial marching is performed using an implicit Euler method. A mapping function is used to transform the [-1 1$]$ Chebyshev domain to an infinite one. Dirichlet boundary conditions are imposed on the flow variables at $r \rightarrow \infty$, and the centreline conditions are dealt with using the procedure described by Mohseni \& Colonius ${ }^{29}$. Initial conditions for the shape functions $\hat{\mathbf{q}}$ are provided by solving the locally parallel, spatial-stability problem using the velocity profile at the first axial station.

\section{Comparison of linear PSE with LES data}

Wave-packet models, obtained with linear PSE, lead to good agreement with experimental results for Strouhal numbers lower than $1 .^{9,10}$ However, it is difficult to obtain measurements for high St resolved into azimuthal modes. A hot-wire sensor used to measure streamwise fluctuations on the jet centreline contains solely the $m=0$ signature and has a low noise level, but this remains too high to sense low-amplitude, high-St wavepackets at that position. A second possibility is to use near-field microphone arrays, but as these should be placed outside the jet it also becomes difficult to accurately measure disturbances related to high-St wavepackets. As will be seen shortly, high-St wavepackets have radial support on a narrow region surrounding the jet lipline, and present a fast exponential decay outside of this region, which lead to the aforementioned experimental difficulties in educing high-St wavepackets.

A different alternative is pursued here, where large-eddy-simulation (LES) data is used. We use the LES of Brès et al. ${ }^{30-32}$ which closely matched experiments of a Mach 0.9 jet in Poitiers by careful computations of the turbulent boundary layer inside the nozzle. In particular, the simulation with 69 million grid points ${ }^{32}$ has shown excellent agreement with the far-field sound up to $\mathrm{St}=4$, which is an important feature for the present study. More details about the LES can be found in the cited papers.

It is important to accurately fit the velocity profiles in the region near the nozzle, as high-St wavepackets depend on the details of the profile due to their relatively small wavelength. To fit the profile shape, we have used an expression similar to the one in Fontaine et al., given, with the present non-dimensional variables, as

$$
U=\frac{M}{4}\left[1-\tanh \left(\frac{r_{o}}{4 \theta_{1}}\left[\frac{r}{r_{o}}-\frac{r_{o}}{r}\right]\right)\right]\left[1-\tanh \left(\frac{r_{o}}{4 \theta_{2}}\left[\frac{r-r_{s}}{r_{o}}-\frac{r_{o}}{r}\right]\right)\right] .
$$

Sample fits of the near-nozzle velocity profiles are shown in fig. 1, showing that the expression represents the data closely.

As in previous works ${ }^{9,10,16}$ a Proper Orthogonal Decomposition (POD) is used to extract the coherent part of velocity and pressure fluctuations. The procedure applied here is similar to Sinha et al., ${ }^{16}$ and further details can be found in that paper. In what follows we will consider the axial velocity $u$ and the pressure $p$ taken from the first POD mode from the LES data (hereafter denoted POD mode 1), which are compared to PSE results. All the results in this paper refer to the axisymmetric mode $(m=0)$.

Figures 2 and 3 shows respectively pressure and axial velocity comparisons between the POD mode 1 from the LES data and the PSE results, here for $\mathrm{St}=0.95$, which is around the maximum value evaluated in previous works. For positions downstream of $x / D=0.4$, up to $x / D=1$, close agreement between LES and PSE is found for both quantities, as in previous works. The typical Kelvin-Helmholtz signature is clearly observed in the first POD mode, with the double bump in the axial velocity which is a sign of a phase jump of $\pi \cdot{ }^{10}$ Some differences appear, however, most prominently for $x / D=0.2$ but also for other positions with $r$ close to 0 . For this Mach 0.9 jet, besides the Kelvin-Helmholtz instability there are other relevant modes, which correspond to trapped acoustic waves in the potential core; further details can be found in Towne et al. ${ }^{33}$ The said acoustic waves are responsible for the radially-oscillatory behaviour seen for both pressure and velocity. As PSE calculations focused on the Kelvin-Helmholtz mode, these low $r$ oscillations are not captured by the model.

Downstream of $x / D=1$ the PSE solutions start to saturate, and then decay slowly. This can be seen in the results for $x / D=2$ and 3. In this decay region, the agreement between PSE and the first POD mode worsens, as previously seen. ${ }^{10}$ This trend is seen for all Strouhal numbers. In what follows, we will focus on the near-nozzle region $x / D \leq 1$, where the Kelvin-Helmholtz wavepackets have spatial amplification.

Similar comparisons are performed for St $=2$ in figs. 4 and 5 . We notice that a good agreement between 

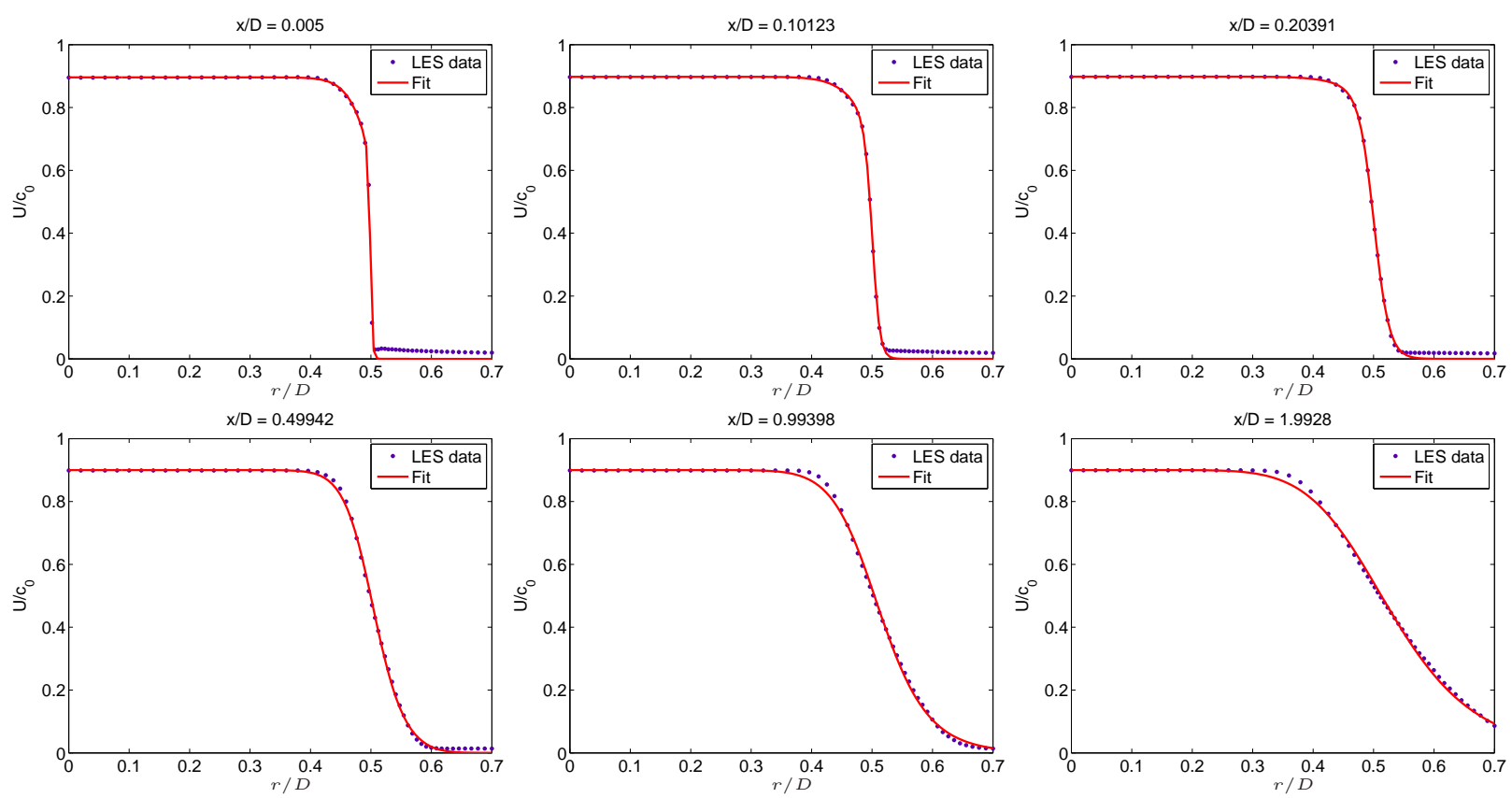

Figure 1. Sample fits of the mean velocity profiles from the LES.
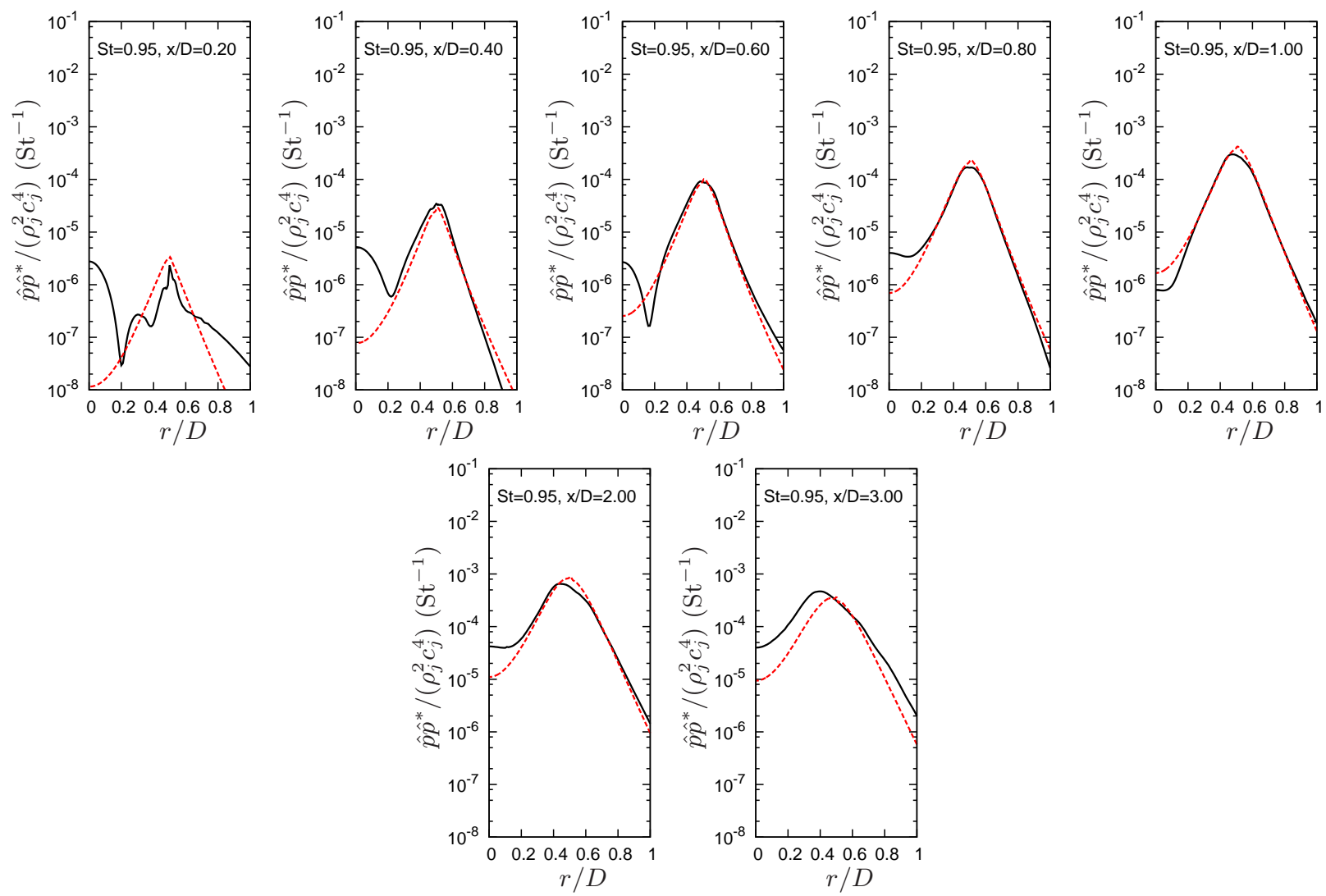

Figure 2. Comparison of pressure from POD mode 1 (full lines) and linear PSE (dashed lines), for St $=0.95$

PSE and POD mode 1 is also seen for this Strouhal number, at least for $0 \leq x / D \leq 1$; the downstream behaviour is similar to what was seen for $\mathrm{St}=0.95$ and is not shown here. Fluctuations become more concentrated around the jet lipline, and both LES and PSE results have sharper radial decays, a feature 

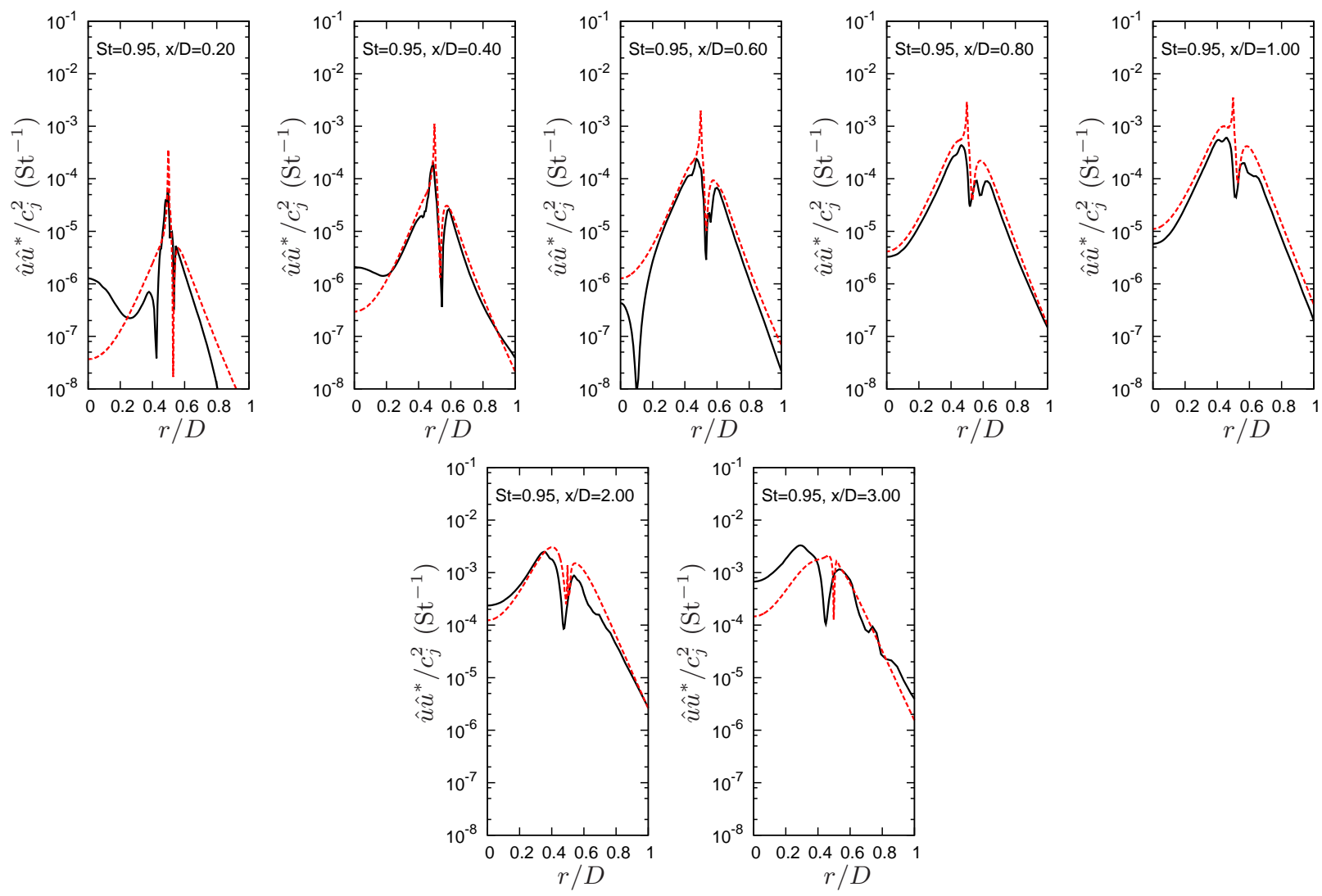

Figure 3. Comparison of axial velocity for POD mode 1 (full lines) and linear PSE (dashed lines), for St $=0.95$

expected from linear stability theory. ${ }^{34}$
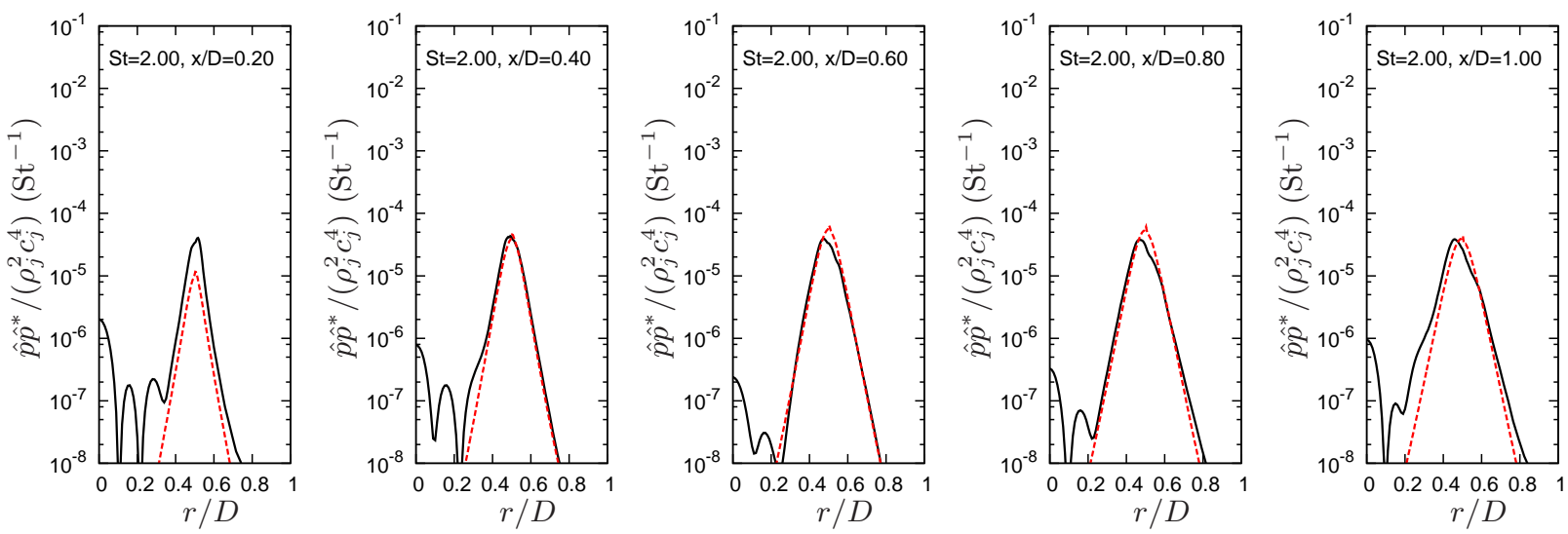

Figure 4. Comparison of pressure from POD mode 1 (full lines) and linear PSE (dashed lines), for St $=2$

Results for St $=3.04$ are shown in figures 6 and 7 . Here the spatial growth of the Kelvin-Helmholtz mode is limited to a region very close to the jet nozzle, and hence results are shown for $x / D=0.1,0.2,0.3$ and 0.4. The last plot is for $x / D=1$ to allow comparisons with results for lower St. We again observe good agreement of PSE with the first POD mode from the LES, and the radial decay outside the lipline is even sharper than for $\mathrm{St}=2$. At the downstream position $x / D=1$ this agreement is already seen to deteriorate.

To present the agreement for several values of $S t$ and $x / D$ in a compact manner, we have calculated the metric

$$
\beta(x, S t)=\frac{\left\langle\hat{u}_{P S E}(x, r, S t), \hat{u}_{P O D}(x, r, S t)\right\rangle}{\left\|\hat{u}_{P S E}(x, r, S t)\right\|\left\|\hat{u}_{P O D}(x, r, S t)\right\|}
$$



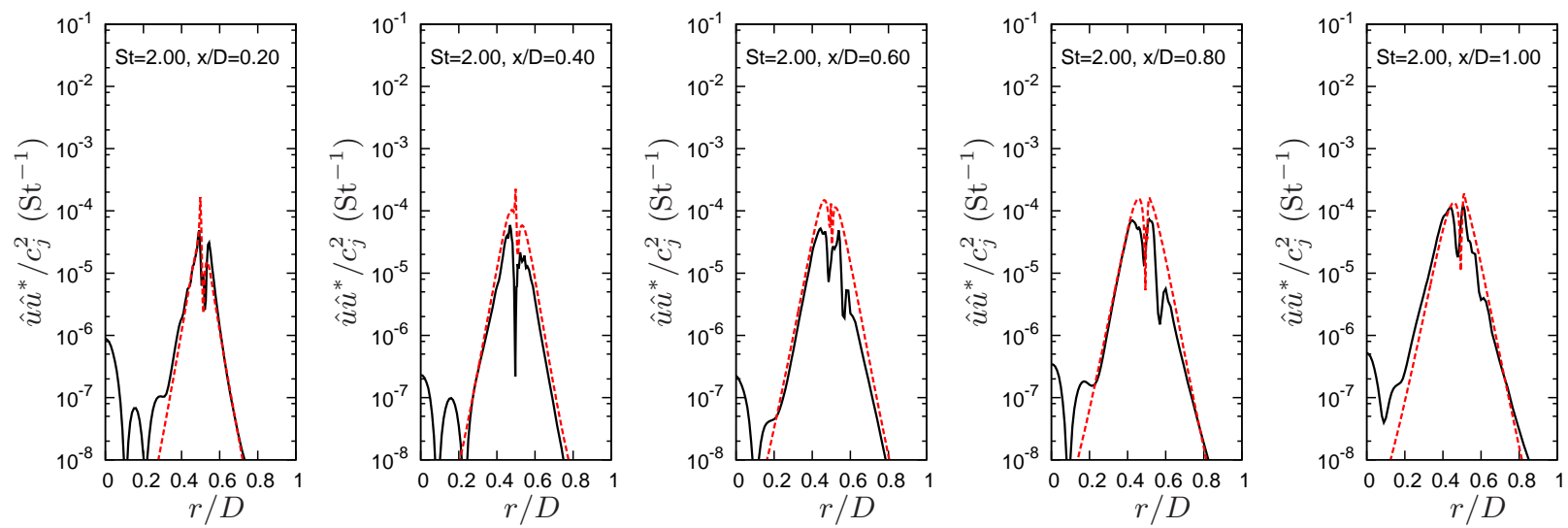

Figure 5. Comparison of axial velocity from POD mode 1 (full lines) and linear PSE (dashed lines), for St $=2$
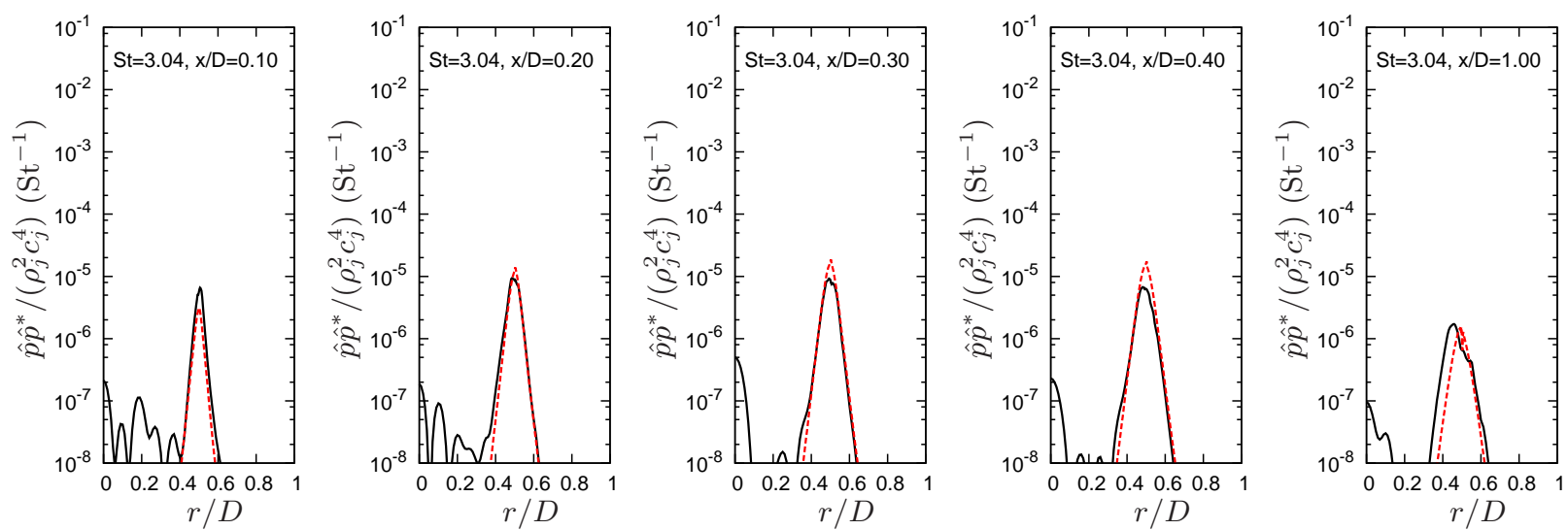

Figure 6. Comparison of pressure from POD mode 1 (full lines) and linear PSE (dashed lines), for St $=3.04$
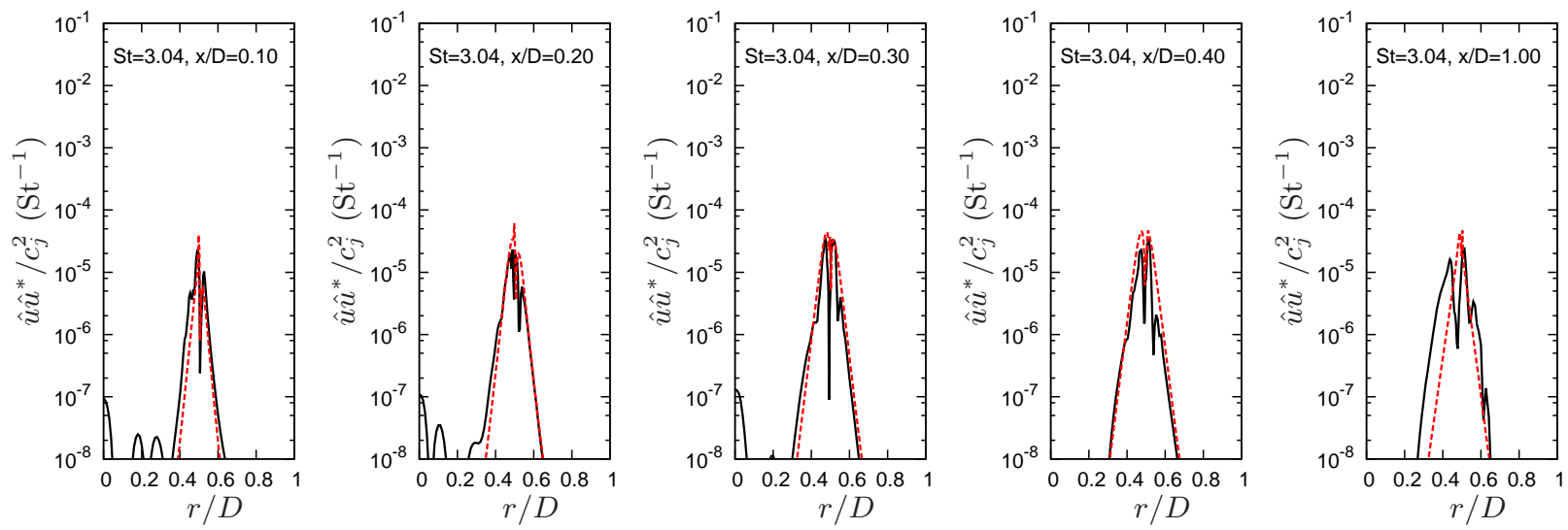

Figure 7. Comparison of pressure from POD mode 1 (full lines) and linear PSE (dashed lines), for St $=3.04$

where the inner product is defined as

$$
\langle f(r), g(r)\rangle=\int_{0}^{\infty} f(r) g^{*}(r) r \mathrm{~d} r .
$$

Perfect agreement for a given $x$, St pair would lead to $\beta=1$, and the other extreme, $\beta=0$, occurs when PSE predictions are orthogonal to the first POD mode. Results are shown in figure 8, where a dashed line indicates the position where $\Im(\alpha)=0$ in the PSE results, which is an indication of the switch between spatial 
growth and decay in wavepackets. We observe that the agreement metric can be as high as 0.9 , particularly for St $<1$, as in our previous study. ${ }^{10}$ However, significant values of $\beta$ are also found for Strouhal numbers of up to 4. The region with good agreement between PSE and the first POD mode is seen to lie upstream of the dashed curve, indicating that the Kelvin-Helmholtz mode has exponential spatial growth in this region. Once it saturates, agreement worsens considerably, leading to $\beta$ values near zero. This is similar to what is observed for $\mathrm{St}<1$, but for higher Strouhal numbers the agreement deteriorates rather fast.

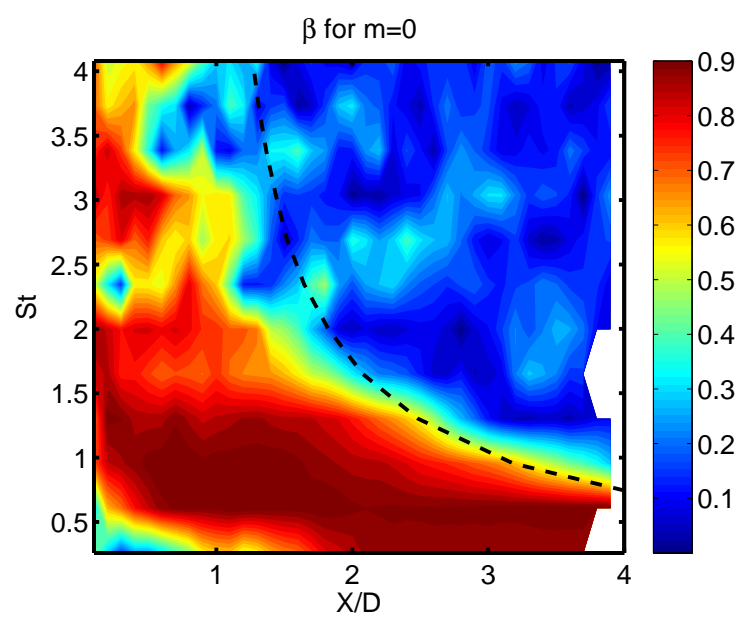

Figure 8. Absolute value of $\beta$ for the axisymmetric mode, between PSE results and POD mode 1 for the axial velocity.

Although not shown here, agreement between PSE and turbulent fluctuations is not good for the axisymmetric mode at Strouhal numbers of about $0.1-0.2$, as in Cavalieri et al. ${ }^{10}$ The $\beta$ values in figure 8 start to have lower values for the lowest St. The reasons for the discrepancies remain unclear, and are the object of ongoing work.

\section{Conclusions}

Detailed comparisons between PSE and LES data (processed by taking its first POD mode) were presented in order to investigate the presence of wavepackets for Strouhal numbers higher than 1 . The availability of a highly-resolved LES, with close agreement to experimental results up to St $=4$, enabled this comparison. The LES data included the nozzle in order to reproduce experimental conditions, where the boundary layer inside the nozzle is tripped such that the shear layers are turbulent from the outset. This allows an investigation of whether the agreement of linear wave-packet models can be extended to higher frequencies, which remain important for the jet noise problem.

Moreover, the capabilities of modelling these high-St behaviour with linear PSE shed light on the role of the upstream boundary layer on jet dynamics and noise. In the simulations of Brès et al. ${ }^{31}$ an accurate model of the turbulent boundary layer inside the nozzle was seen to be important for accurate jet noise predictions. When the jet LES was carried out in a configuration where the boundary layer in the nozzle was laminar, overpredictions of the radiated sound for St $>1$ were observed. The thickness of an initially laminar shear layer increases slowly, due to viscous diffusion, in contrast with the much faster spread of a turbulent shear layer. These low thicknesses of laminar shear layers should lead to more unstable high-St wavepackets, which could explain the overpredictions of sound radiation. Further work on that matter appears promising.

The results presented here show that wavepacket models represent accurately the near-nozzle dynamics for up to $\mathrm{St} \approx 4$. All these results are for the axisymmetric, $m=0$ mode. Although not shown here, similar results were obtained for modes $m=1,2,3$ and 4 . The spatial growth of disturbances in the near-nozzle region can thus be modelled as a linear process, with a linearisation taken around the turbulent mean flow, even for relatively high Strouhal numbers and azimuthal wavenumbers. For downstream positions, linearised models predict a decay of wavepackets, which is not observed in experiments or simulations. This difference can be attributed to non-linearity, where wavepackets are forced by other turbulent fluctuations. ${ }^{35}$ Linear models can be potentially enhanced to include stochastic forcing of background turbulent disturbances, as in Towne et al. ${ }^{36}$ 


\section{Acknowledgments}

AVGC and PJ acknowledge support from the Science Without Borders program (project number A073/2013). AVGC was supported by a CNPq research scholarship, and by CNPq grant 444796/2014-2. AT and TC gratefully acknowledge support from the Office of Naval Research under contract N0014-11- 1-0753. The LES studies are supported by NAVAIR SBIR project, under the supervision of Dr. John T. Spyropoulos. The main calculations were carried out on CRAY XE6 machines at DoD supercomputer facilities in ERDC DSRC.

\section{References}

${ }^{1}$ Bowes, W., Bowler, D., Carnes, R., Fratarangelo, R., Rumpf, D., Heiser, W. H., Huff, D. L., and Moin, P., "Report on jet noise reduction," Tech. rep., Naval Research Advisory Committee, 2009.

${ }^{2}$ Sasaki, K., Estudo e controle de pacotes de onda em jatos utilizando as equações de estabilidade parabolizadas (in portuguese), Master's thesis, ITA - Instituto Tecnológico de Aeronáutica, São José dos Campos, 2014.

${ }^{3}$ Rodrıguez, D., Sinha, A., Bres, G. A., and Colonius, T., "Acoustic field associated with parabolized stability equation models in turbulent jets," 2013.

${ }^{4}$ Jordan, P. and Colonius, T., "Wave packets and turbulent jet noise," Annual Review of Fluid Mechanics, Vol. 45, 2013, pp. $173-195$.

${ }^{5}$ Crighton, D. and Gaster, M., "Stability of slowly diverging jet flow," J. Fluid Mech, Vol. 77, No. 2, 1976, pp. $387-413$.

${ }^{6}$ Cohen, J. and Wygnanski, I., "The evolution of instabilities in the axisymmetric jet. Part 1. The linear growth of disturbances near the nozzle," Journal of Fluid Mechanics, Vol. 176, 1987, pp. 191-219.

${ }^{7}$ Petersen, R. and Samet, M., "On the preferred mode of jet instability," Journal of Fluid Mechanics, Vol. 194, 1988, pp. $153-173$.

${ }^{8}$ Suzuki, T. and Colonius, T., "Instability waves in a subsonic round jet detected using a near-field phased microphone array," Journal of Fluid Mechanics, Vol. 565, 2006, pp. 197-226.

${ }^{9}$ Gudmundsson, K. and Colonius, T., "Instability wave models for the near-field fluctuations of turbulent jets," Journal of Fluid Mechanics, Vol. 689, 2011, pp. 97-128.

${ }^{10}$ Cavalieri, A. V. G., Rodríguez, D., Jordan, P., Colonius, T., and Gervais, Y., "Wavepackets in the velocity field of turbulent jets," Journal of Fluid Mechanics, Vol. 730, 2013, pp. 559-592.

${ }^{11}$ Léon, O. and Brazier, J.-P., "Application of the Linear Parabolized Stability Equations to a Subsonic Coaxial Jet," 17th AIAA/CEAS Aeroacoustics Conference (32nd AIAA Aeroacoustics Conference), 2011.

${ }^{12}$ Gloor, M., Obrist, D., and Kleiser, L., "Linear stability and acoustic characteristics of compressible, viscous, subsonic coaxial jet flow," Physics of Fluids (1994-present), Vol. 25, No. 8, 2013, pp. 084102.

${ }^{13}$ Malik, M. and Chang, C.-L., "Nonparallel and nonlinear stability of supersonic jet flow," Computers 6 fluids, Vol. 29, No. 3, 2000, pp. 327-365.

${ }^{14}$ Nichols, J. W. and Lele, S. K., "Global modes and transient response of a cold supersonic jet," Journal of Fluid Mechanics, Vol. 669, 2011, pp. 225-241.

${ }^{15}$ Ray, P. K. and Lele, S. K., "Sound generated by instability wave/shock-cell interaction in supersonic jets," Journal of Fluid Mechanics, Vol. 587, 2007, pp. 173-215.

${ }^{16}$ Sinha, A., Rodríguez, D., Brès, G. A., and Colonius, T., "Wavepacket models for supersonic jet noise," Journal of Fluid Mechanics, Vol. 742, 2014, pp. 71-95.

${ }^{17}$ Crighton, D. and Huerre, P., "Shear layer pressure fluctuations and superdirective acoustic sources," Journal of Fluid Mechanics, Vol. 220, 1990, pp. 355-368.

${ }^{18}$ Cavalieri, A. V. and Agarwal, A., "Coherence decay and its impact on sound radiation by wavepackets," Journal of Fluid Mechanics, Vol. 748, 2014, pp. 399-415.

${ }^{19}$ Avital, E. and Sandham, N., "A note on the structure of the acoustic field emitted by a wave packet," Journal of sound and vibration, Vol. 204, No. 3, 1997, pp. 533-539.

${ }^{20}$ Obrist, D., "Directivity of acoustic emissions from wave packets to the far field," Journal of Fluid Mechanics, Vol. 640, 2009, pp. 165-186.

${ }^{21}$ Papamoschou, D., "Wavepacket modeling of the jet noise source," AIAA Paper, Vol. 2835, 2011, pp. 2011.

${ }^{22}$ Garnaud, X., Sandberg, R. D., and Lesshafft, L., "Global response to forcing in a subsonic jet: instability wavepackets and acoustic radiation," .

${ }^{23}$ Salgado, A. M., Agarwal, A., Suponitsky, V., and Sandham, N. D., "A non-linear PSE-based model for jet noise," AIAA Paper 2010, Vol. 3969, 2010.

${ }^{24}$ Cheung, L. C., Bodony, D. J., and Lele, S. K., "Noise radiation predictions from jet instability waves using a hybrid nonlinear PSE-acoustic analogy approach," Proc. 13th AIAA/CEAS Aeroacoustics Conf., Rome, Italy, 21-23 May $2007,2007$.

${ }^{25}$ Sandham, N. and Salgado, A., "Nonlinear interaction model of subsonic jet noise," Philosophical Transactions of the Royal Society A: Mathematical, Physical and Engineering Sciences, Vol. 366, No. 1876, 2008, pp. 2745-2760.

${ }^{26}$ Harper-Bourne, M., "Jet noise measurements: past and present," International Journal of Aeroacoustics, Vol. 9, No. 4, 2010, pp. 559-588.

${ }^{27}$ Fontaine, R. A., Elliott, G. S., Austin, J. M., and Freund, J. B., "Very near-nozzle shear-layer turbulence and jet noise." Journal of Fluid Mechanics, Vol. 770, 2015, pp. 27-51.

${ }^{28}$ Herbert, T., "Parabolized stability equations," Annual Review of Fluid Mechanics, Vol. 29, No. 1, 1997, pp. $245-283$. 
${ }^{29}$ Mohseni, K. and Colonius, T., "Numerical treatment of polar coordinate singularities," Journal of Computational Physics, Vol. 157, No. 2, 2000, pp. 787-795.

${ }^{30}$ Brès, G. A., Jordan, P., Colonius, T., Le Rallic, M., Jaunet, V., and Lele, S. K., "Large eddy simulation of a Mach 0.9 turbulent jet," Proceedings of the Summer Program, Center for Turbulence Research, Stanford University, 2014.

${ }^{31}$ Brès, G. A., Jaunet, V., Le Rallic, M., Jordan, P., Colonius, T., and Lele, S. K., "Large eddy simulation for jet noise: the importance of getting the boundary layer right," AIAA paper 2015-2535, 2015.

${ }^{32}$ Brès, G. A. et al., "Large eddy simulation for jet noise: azimuthal decomposition and intermittency of the radiated sound," 22th AIAA/CEAS Aeroacoustics Conference, 2016.

${ }^{33}$ Towne, A., Cavalieri, A. V. G., Jordan, P., Colonius, T., Jaunet, V., Schmidt, O., and Brès, G., "Trapped acoustic waves in the potentia core of subsonic jets," 22th AIAA/CEAS Aeroacoustics Conference, 2016.

${ }^{34}$ Michalke, A., "Survey on jet instability theory," Progress in Aerospace Sciences, Vol. 21, 1984, pp. 159-199.

${ }^{35}$ Tissot, G., Zhang, M., Lajús Jr, F. C., Cavalieri, A. V. G., Jordan, P., and Colonius, T., "Sensitivity of wavepackets in jets to non-linear effects: the role of the critical layer," 21th AIAA/CEAS Aeroacoustics Conference, 2015.

${ }^{36}$ Towne, A., Colonius, T., Jordan, P., Cavalieri, A., and Brès, G. A., "Stochastic and nonlinear forcing of wavepackets in a Mach 0.9 jet," 21st AIAA/CEAS Aeroacoustics Conference, 2015, p. 2217. 